\title{
TENDENCIA DE LOS ARTÍCULOS DE PSICOLOGÍA CLÍNICA EN CHILE, LATINOAMÉRICA Y ESPAÑA
}

TENDENCIES IN CLINICAL PSYCHOLOGY ARTICLES IN CHILE, LATIN-AMERICAN AND SPAIN

Recibido: 28 de Marzo del 2011 | Aceptado: 28 de Abril del 2011

GERARDO RIFFO ALLENDE

(UNIVERSIDAD SANTIAGO DE CHILE, Región Metropolitana, Chile).

\begin{abstract}
RESUMEN
El objetivo de este estudio ex post facto historiográfico bibliométrico es describir la tendencia de los artículos en Psicología Clínica de Chile, Latinoamérica y España entre 2004 y 2010 a partir de los artículos de la revista Terapia psicológica, Revista Latinoamérica de Psicología y Revista Internacional de Psicología Clínica y de la Salud/ International Journal of Clinical and Health Psychology. El número de publicaciones en Terapia psicológica es de 112, en la revista Internacional de Psicología Clínica y de la Salud/ International Journal of Clinical and Health Psychology es de 200 y en la Revista Latinoamérica de Psicología se encuentran 143 artículos del ámbito clínico. Dentro de este periodo destacan los artículos centrados en la psicopatología, la evaluación psicológica y la psicoterapia mientras que las áreas menos abordadas son el diagnóstico clínico y la persona del terapeuta. El enfoque terapéutico de mayor interés fue el cognitivo conductual.
\end{abstract}

PALABRAS CLAVE: Estudio ex post facto, Estudio Bibliométrico, Psicología Clínica.

\section{ABSTRACT}

The objective of this ex post facto bibliometric historiography study is to describe the tendency in Clinical Psychology Articles in Chile, Latin America and Spain between 2004 and 2010 from articles of Terapia psicológica, Revista Latinoamérica de Psicología y Revista Internacional de Psicología Clínica y de la Salud/ International Journal of Clinical and Health Psychology journals. The number of publications in Terapia psicológica is 112, in Internacional de Psicología Clínica y de la Salud/ International Journal of Clinical and Health Psychology is 200 and in Revista Latinoamérica de Psicología there are 143 articles in clinical settings. In this period it can be distinguished articles focused on psychopathology, psychological assessment and psychotherapy while the least addressed areas are the clinical diagnosis and the person of the therapist. The therapeutic focus of interest was the cognitive behaviour.

KEY WORDS: Ex post facto study, Bibliometric study, Clinical psychology.

1. Gerardo Riffo Allende . Dirección: Ecuador 3363, Estación Central, Región Metropolitana, Chile.

Correo: gerardo_riffo@yahoo.es 
INTRODUCCIÓN

La literatura científica en psicología al igual que en la mayoría de las ciencias se pueden encontrar ampliamente difundidas en artículos de revistas científicas especializadas. Estas revistas tienen una amplia variedad de funciones dentro de la comunidad científica, son un reflejo en el desarrollo de una disciplina como lo señala Alcain y Lascurain (2002) y son indicadores del estado de la producción científica para cualquier país o zona geográfica (Agudelo, Bretón-López y Buela-Casal, 2003).

El interés por la producción científica como un índice de calidad y para describir y analizar el desarrollo de una disciplina científica ha dado pie al crecimiento y fortalecimiento de los estudios bibliométricos, los cuales abordan esta temática en cuanto a datos cuantitativos. Autores como Buela-Casal, Carretero-Dios y De los Santos-Roig (2002) señalan que no deben ser vistos como los únicos criterios de evaluación de la calidad. Estos estudios tienen un valor dentro de la literatura científica ya que orientan al lector en torno a asuntos académicos tales como las principales temáticas que se abordan en ellas, el índice de autoría o las citas que reciben determinados artículos (Agudelo et al. 2003). Pese a esto, diversos autores indican que los estudios bibliométricos presentan dificultades y errores metodológicos y resultan ser bastantes controvertidos. Así lo señala Buela-Casal $(2001,2002,2003)$ y Pelechano (2002).

En nuestro país la evaluación de la producción científica en esta área es insuficiente, artículos referidos al estado del arte de la psicología clínica y de su medición pueden verse en Aceituno, Krause y Winkler (2000), Moyano y Ramos (2000), Vera-Villarroel y Lillo (2006), como también análisis sobre las intervenciones apoyadas empíricamente en Ortiz y Vera-Villarroel (2003).
A nivel latinoamericano es posible encontrar mayores sistemas de evaluación y medición. Esto producto de congresos y simposios centrados en aumentar la visibilidad de las revistas iberoamericanas de psicología (Buela-Casal, Carretero-Dios y De los Santos-Roig, 2002). Artículos orientados en este ámbito pueden verse en Rey, Martínez y Rodríguez (2009), BuelaCasal y Lopez (2005), Agudelo et al. (2003), Alcain y Lascurain (2002) y Perdomo, Zambrano, López y Pérez (2003).

En España la situación es distinta, ya que cuentan con el índice de impacto de las revistas españolas de ciencias sociales (INRECS) que es un índice bibliométrico que ofrece información estadística con el fin de determinar la relevancia, influencia e impacto científico de las revistas españolas de ciencias sociales. Además cuentan con numerosos estudios acerca del estado actual de la psicología en España y variados análisis bibliométricos, entre los que destacan Pérez (2002), Alcain y Carpintero (2001), Buela-Casal, Sierra, Carretero y Santos (2002), Jiménez-Contreras, Faba y Moya (2001) y Civera y Tortosa (2001).

En este sentido cabe la necesidad de evaluar el estado del arte de la psicología clínica de nuestro país frente a Latinoamérica y España, para así conocer y comparar las principales temáticas y tendencias que se están abordando. De esta manera, el objetivo del presente estudio es hacer un análisis bibliométrico de tres revistas de psicología clínica y contrastar dicha información obtenida.

\section{MÉTODO}

\section{Unidades de análisis}

Para este estudio ex post facto historiográfico bibliométrico (Montero y León, 2007) se realizó una búsqueda de los artículos publicados entre el 2004 y 2010 de las revistas Terapia Psicológica, Revista Latinoamericana de Psicología y Revista Internacional de Psicología Clínica y de la 
Salud/ International Journal of Clinical and Health Psychology, cuyo texto completo o resumen se podía recuperar a través del sistema de información Redalyc.

Se escogieron debido a que son publicaciones de acceso libre que abordan el área de la psicología clínica, son revistas de larga data que desde sus inicios se publican sin interrupciones y están incluidas en diversas bases de datos contribuyendo en el desarrollo y la divulgación de la producción académica en estas regiones. Estudios previos y similares pueden verse en Agudelo et al. (2003), Buela-Casal y López (2005), Rey, Martínez y Guerrero (2009) y Calvache, López y Mayorga (2002).

\section{Materiales}

Terapia Psicológica (ISSN: 0716-6184) es una revista fundada por la Sociedad Chilena de Psicología Clínica en el año 1982, se divulga sin interrupciones hasta la fecha y está orientada a promover el intercambio de conocimientos que aporten en la psicología científica, estimulando la crítica dentro de la disciplina, de acuerdo a la discusión de datos, metodología, planteamientos teóricos e integración multidisciplinaria. Se publican dos números al año y acepta trabajos en castellano e inglés.

Revista Latinoamericana de Psicología (ISSN: 0120-0534) publicada por primera vez en 1969, fue fundada por el psicólogo Rubén Ardila y a partir del 2007 comienza a ser publicada por la Fundación Universitaria Konrad Lorenz. Se trata de una revista que publica trabajos empíricos y teóricos, en el abordaje de problemas de carácter básico y aplicado, en todas las áreas de la psicología. Se publican tres números al año y está incluida en las principales bases de datos a nivel internacional, siendo considerada la principal revista de psicología en Iberoamérica.
Revista Internacional de Psicología Clínica y de la Salud / International Journal of Clinical and Health Psychology (ISSN: 1576-7329) es una revista editada por la Asociación Española de Psicología Conductual desde el año 2001 y cuenta con la colaboración de la Asociación Colombiana para el Avance de las Ciencias del Comportamiento y la associação Portuguesa de Terapia do Comportamento. En esta revista se publican trabajos de carácter básico y aplicado y tanto teóricos como experimentales que contribuyen al avance en el ámbito de la psicología clínica y de la salud. Se publican tres números al año y acepta trabajos en castellano, inglés y portugués.

\section{Procedimiento}

El procedimiento de obtención de datos fue realizado en varias etapas. A partir de las revistas mencionadas anteriormente se seleccionaron los artículos de Psicología Clínica entre los años 2004 y 2010.

Dentro de las revistas utilizadas se consideraran todos los artículos originales de las revistas Terapia Psicológica y Revista Internacional de Psicología Clínica y de la Salud. Por otra parte, la revista Latinoamericana de Psicología tiene artículos de diversas áreas de la psicología, por lo que, para el presente estudio serán considerados sólo los artículos originales que tengan relación con la psicología clínica, considerando estos por medio de una búsqueda exhaustiva en los campos "título", "resumen" y "palabras clave".

En base a estudios similares, (Agudelo et al. 2003 y Rey et al. 2009) se considerará una clasificación de los artículos de psicología clínica en: diagnóstico psicológico; evaluación psicológica; persona del terapeuta; prevención y promoción de la salud; psicopatología; enfoque terapéutico e intervención/psicoterapia, registrándose el número de artículos que se encontraba en cada una de ellas. Esto se ha realizado de modo excluyente, incluyendo a cada artículo 
en la categoría más afín aunque guardara relación con alguna otra.

Para el caso del enfoque terapéutico, se realizó una subdivisión para realizar un análisis más exhaustivo dividiéndose en: terapia psicoanalítica; terapia cognitiva conductual; terapia humanista y terapia sistémica, ya que, investigaciones las señalan como los principales enfoques terapéuticos en la actualidad (Trull y Phares, 2003).

El índice de autoría se consideró en función del número de firmantes por artículo y a partir de esto, se pueden clasificar en artículos de autoría individual, autoría de pareja y en artículos de autoría compartida.

El análisis de las revistas se realizó por parte de dos investigadores de forma individual y después se calculó el acuerdo de los datos obtenidos para identificar el grado de acuerdo-desacuerdo, hasta encontrar un acuerdo en función de los criterios establecidos. Cabe señalar que la búsqueda se realizó en los meses de marzo y abril de 2011 y el porcentaje de acuerdos en todos los índices y mediciones fue superior al $92 \%$.

\section{RESULTADOS}

Distribución de artículos de psicología clínica publicados por año

Durante el periodo analizado entre el año 2004 y 2010 en la revista Terapia Psicológica se han publicado 112 artículos originales (véase la tabla 1). En la Revista Latinoamericana de Psicología se han publicado 224 artículos, de los cuales 143 pertenecen al ámbito clínico específicamente (véase la tabla 2) y en la Revista Internacional de Psicología Clínica y de la Salud se han publicado 200 artículos (véase la tabla 3) y a fin de tener una visión más detallada de lo que sucede en estas tres revistas frente a la distribución de artículos publicados por año (véase Figura 1).
TABLA 1.

Distribución de artículos según año en la Revista Terapia Psicológica entre 2004 y 2010

\begin{tabular}{ccc}
\hline Año & $\begin{array}{c}\text { Número de } \\
\text { artículos }\end{array}$ & $\%$ \\
\hline 2004 & 13 & 11,61 \\
2005 & 13 & 11,61 \\
2006 & 15 & 13,39 \\
2007 & 17 & 15,18 \\
2008 & 16 & 14,29 \\
2009 & 20 & 17,85 \\
2010 & 18 & 16,07 \\
Total & 112 & 100,0 \\
\hline
\end{tabular}

TABLA 2.

Distribución de artículos de Psicología Clínica según año en la Revista Latinoamericana de Psicología entre 2004 y 2010.

\begin{tabular}{ccc}
\hline Año & $\begin{array}{c}\text { Número de } \\
\text { artículos }\end{array}$ & $\%$ \\
\hline 2004 & 18 & 12,59 \\
2005 & 20 & 13,99 \\
2006 & 19 & 13,29 \\
2007 & 22 & 15,38 \\
2008 & 23 & 16,08 \\
2009 & 20 & 13,99 \\
2010 & 21 & 14,68 \\
Total & 143 & 100,0 \\
\hline
\end{tabular}

TABLA 3.

Distribución de artículos según año en la Revista Internacional de Psicología Clínica y de la Salud entre 2004 y 2010.

\begin{tabular}{ccc}
\hline Año & $\begin{array}{c}\text { Número de } \\
\text { artículos }\end{array}$ & $\%$ \\
\hline 2004 & 24 & 12,00 \\
2005 & 18 & 09,00 \\
2006 & 29 & 14,50 \\
2007 & 36 & 18,00 \\
2008 & 39 & 19,50 \\
2009 & 28 & 14,00 \\
2010 & 26 & 13,00 \\
Total & 200 & 100,0 \\
\hline
\end{tabular}




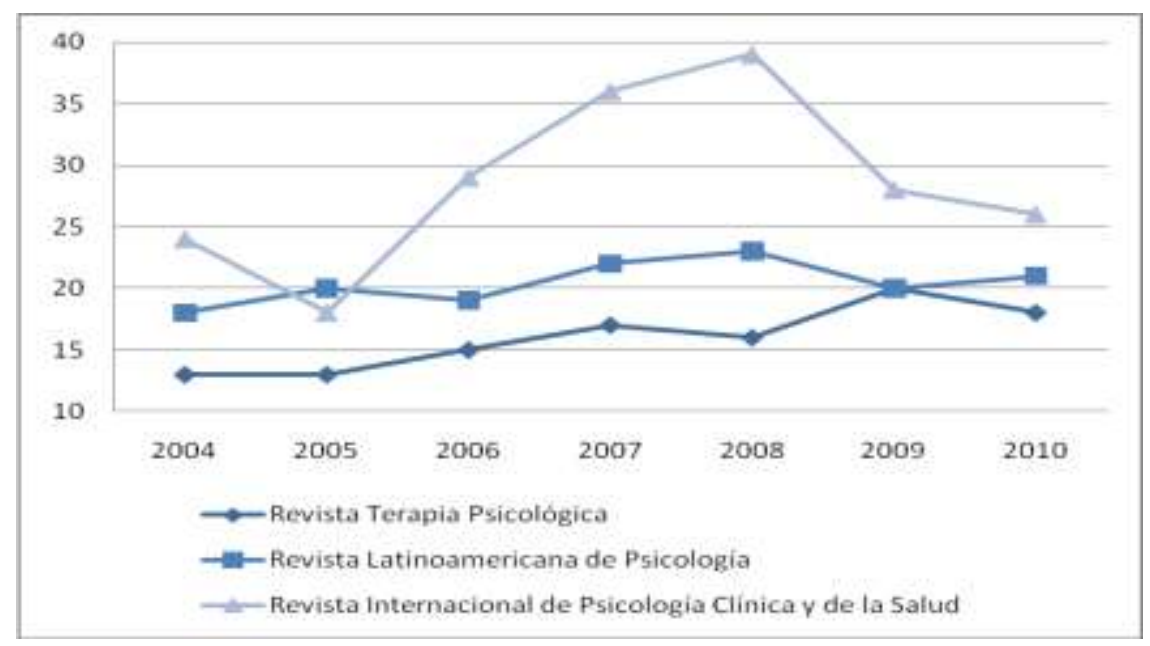

FIGURA 1. Distribución de artículos de Psicología Clínica publicadas entre el 2004 y 2010.

Distribución de artículos de psicología clínica según el número de autores

En relación al número de autores que firman los trabajos la mayoría de los artículos publicados en la revista Terapia Psicológica $50 \%$ están publicados por 1 y 2 autores (véase la tabla 4), la mayoría de los artículos publicados en la Revista Latinoamericana de Psicología 55,95\% están publicados por 2 y 3 autores (véase la tabla 5) y la mayoría de los artículos publicados en la Revista Internacional de Psicología Clínica y de la Salud $52 \%$ están publicados por 3 y 4 autores (véase la tabla $6)$.

\section{TABLA 4.}

Distribución de artículos según el número de autores en la Revista Terapia Psicológica entre 2004 y 2010

\begin{tabular}{ccc}
\hline $\begin{array}{c}\mathrm{N}^{\circ} \text { de autores por } \\
\text { articulo }\end{array}$ & $\begin{array}{c}\mathrm{N}^{\circ} \text { de } \\
\text { artículos }\end{array}$ & $\%$ \\
\hline 1 & 23 & 20,54 \\
2 & 33 & 29,46 \\
3 & 17 & 15,18 \\
4 & 16 & 14,29 \\
5 & 11 & 09,82 \\
6 & 7 & 06,25 \\
7 & 1 & 00,89 \\
8 & 2 & 01,79 \\
9 & 1 & 00,89 \\
10 & 1 & 00,89 \\
Total & 112 & 100,0 \\
\hline
\end{tabular}

TABLA 5.

Distribución de artículos de Psicología Clínica según el número de autores en la Revista Latinoamericana de Psicología entre 2004 y 2010.

\begin{tabular}{ccc}
\hline $\begin{array}{c}\mathrm{N}^{\circ} \text { de autores por } \\
\text { articulo }\end{array}$ & $\begin{array}{c}\mathrm{N}^{\circ} \text { de } \\
\text { artículos }\end{array}$ & $\%$ \\
\hline 1 & 25 & 17,48 \\
2 & 43 & 30,08 \\
3 & 37 & 25,87 \\
4 & 23 & 16,08 \\
5 & 9 & 06,29 \\
6 & 3 & 02,10 \\
7 & 2 & 01,40 \\
8 & 1 & 00,70 \\
9 & 0 & 00,00 \\
10 & 0 & 00,00 \\
Total & 143 & 100,0 \\
\hline
\end{tabular}

\section{TABLA 6.}

Distribución de artículos según el número de autores en la Revista Internacional de Psicología Clínica y de la Salud entre 2004 y 2010

\begin{tabular}{ccc}
\hline $\begin{array}{c}\mathrm{N}^{\circ} \text { de autores por } \\
\text { articulo }\end{array}$ & $\begin{array}{c}\mathrm{N}^{\circ} \text { de } \\
\text { artículos }\end{array}$ & $\%$ \\
\hline 1 & 7 & 03,50 \\
2 & 36 & 18,00 \\
3 & 63 & 31,50 \\
4 & 41 & 20,50 \\
5 & 24 & 12,00 \\
6 & 17 & 08,50 \\
7 & 4 & 02,00 \\
8 & 5 & 02,50 \\
9 & 2 & 01,00 \\
10 & 1 & 00,50 \\
Total & 200 & 100,0 \\
\hline
\end{tabular}


Distribución de artículos de psicología clínica según área de conocimiento

En relación a la distribución de los artículos según el área de conocimiento en la revista Terapia Psicológica, estos se concentran en la evaluación psicológica psicopatología y la intervención/psicoterapia, y dentro del área menos abordada, el diagnóstico psicológico (véase la tabla 7). En la Revista Latinoamericana de Psicología, estos se concentran en la psicopatología y en la prevención y promoción, y el área menos abordada es la persona del terapeuta (véase la tabla 8). En la Revista Internacional de Psicología Clínica y de la Salud, estos se concentran en la psicopatología y la evaluación psicológica siendo el área menos abordada la persona del terapeuta (véase la tabla 9).

TABLA 7.

Distribución de artículos según el área de conocimiento en la Revista Terapia Psicológica entre 2004 y 2010

\begin{tabular}{lcccccccc}
\hline \multicolumn{1}{c}{ Área de conocimiento } & 2004 & 2005 & 2006 & 2007 & 2008 & 2009 & 2010 & Total \\
\hline Diagnostico Psicológico & 1 & 0 & 1 & 1 & 0 & 1 & 0 & 4 \\
Evaluación Psicológica & 3 & 3 & 3 & 5 & 5 & 5 & 9 & 33 \\
Prevención y Promoción & 1 & 1 & 4 & 2 & 3 & 4 & 1 & 16 \\
Persona del terapeuta & 0 & 2 & 0 & 1 & 2 & 2 & 0 & 7 \\
Psicopatología & 3 & 2 & 3 & 3 & 3 & 3 & 1 & 18 \\
Enfoque terapéutico & 1 & 2 & 2 & 1 & 1 & 3 & 1 & 11 \\
Intervención/Psicoterapia & 4 & 3 & 2 & 4 & 2 & 2 & 6 & 23 \\
Total & 13 & 13 & 15 & 17 & 16 & 20 & 18 & 112 \\
\hline
\end{tabular}

TABLA 8

Distribución de artículos según el área de conocimiento en la Revista Latinoamericana de Psicología entre 2004 y 2010

\begin{tabular}{lcccccccc}
\hline \multicolumn{1}{c}{ Área de conocimiento } & 2004 & 2005 & 2006 & 2007 & 2008 & 2009 & 2010 & Total \\
\hline Diagnostico Psicológico & 1 & 2 & 1 & 1 & 2 & 2 & 1 & 10 \\
Evaluación Psicológica & 3 & 5 & 3 & 5 & 4 & 3 & 7 & 30 \\
Prevención y Promoción & 3 & 4 & 4 & 5 & 6 & 5 & 5 & 32 \\
Persona del terapeuta & 0 & 0 & 0 & 0 & 1 & 0 & 0 & 1 \\
Psicopatología & 5 & 4 & 4 & 5 & 5 & 6 & 3 & 32 \\
Enfoque terapéutico & 1 & 2 & 3 & 2 & 1 & 2 & 1 & 12 \\
Intervención/Psicoterapia & 5 & 3 & 4 & 4 & 4 & 2 & 4 & 26 \\
Total & 18 & 20 & 19 & 22 & 23 & 20 & 21 & 143 \\
\hline
\end{tabular}

TABLA 9

Distribución de artículos según el área de conocimiento en la Revista Latinoamericana de Psicología entre 2004 y 2010.

\begin{tabular}{lcccccccc}
\hline \multicolumn{1}{c}{ Área de conocimiento } & 2004 & 2005 & 2006 & 2007 & 2008 & 2009 & 2010 & Total \\
\hline Diagnostico Psicológico & 1 & 0 & 1 & 1 & 2 & 0 & 4 & 9 \\
Evaluación Psicológica & 4 & 3 & 6 & 9 & 8 & 8 & 10 & 48 \\
Prevención y Promoción & 3 & 1 & 5 & 6 & 7 & 6 & 3 & 31 \\
Persona del terapeuta & 0 & 0 & 0 & 0 & 0 & 0 & 1 & 1 \\
Psicopatología & 6 & 5 & 7 & 8 & 9 & 6 & 4 & 45 \\
Enfoque terapéutico & 4 & 6 & 4 & 4 & 5 & 4 & 2 & 29 \\
Intervención/Psicoterapia & 6 & 3 & 6 & 8 & 8 & 4 & 2 & 37 \\
Total & 24 & 18 & 29 & 36 & 39 & 28 & 26 & 200 \\
\hline
\end{tabular}


Distribución de artículos de psicología clínica según enfoque terapéutico

En relación a la distribución de los artículos según el enfoque terapéutico, en la revista Terapia Psicológica, estos se concentran en el enfoque cognitivo conductual y se encuentran artículos en el enfoque sistémico y psicoanalítico (véase la tabla 10). En la Revista Latinoamericana de
Psicología, estos se concentran en el enfoque cognitivo conductual y en el enfoque sistémico (véase la tabla 11). En la Revista Internacional de Psicología Clínica y de la Salud, estos se concentran en el enfoque cognitivo conductual, sin encontrarse artículos desde otro enfoque terapéutico (véase la tabla 12).

TABLA 10.

Distribución de artículos según Enfoque Terapéutico en la Revista Terapia Psicológica entre 2004 y 2010.

\begin{tabular}{lcccccccc}
\hline \multicolumn{1}{c}{ Enfoque Terapéutico } & 2004 & 2005 & 2006 & 2007 & 2008 & 2009 & 2010 & Total \\
\hline Enfoque Psicoanalítica & 0 & 1 & 0 & 0 & 0 & 0 & 0 & 1 \\
Enfoque Cognitivo conductual & 1 & 1 & 1 & 1 & 1 & 2 & 1 & 8 \\
Enfoque Humanista & 0 & 0 & 0 & 0 & 0 & 0 & 0 & 0 \\
Enfoque Sistémico & 0 & 0 & 1 & 0 & 0 & 1 & 0 & 2 \\
Total & 1 & 2 & 2 & 1 & 1 & 3 & 1 & 11 \\
\hline
\end{tabular}

TABLA 11.

Distribución de artículos según Enfoque Terapéutico en la Revista Latinoamericana de Psicología entre 2004 y 2010

\begin{tabular}{lcccccccc}
\hline \multicolumn{1}{c}{ Enfoque Terapéutico } & 2004 & 2005 & 2006 & 2007 & 2008 & 2009 & 2010 & Total \\
\hline Enfoque Psicoanalítica & 0 & 0 & 0 & 0 & 0 & 0 & 0 & 0 \\
Enfoque Cognitivo conductual & 1 & 2 & 2 & 1 & 1 & 2 & 1 & 9 \\
Enfoque Humanista & 0 & 0 & 0 & 0 & 0 & 0 & 0 & 0 \\
Enfoque Sistémico & 0 & 0 & 1 & 1 & 0 & 0 & 0 & 2 \\
Total & 1 & 2 & 3 & 2 & 1 & 2 & 1 & 12 \\
\hline
\end{tabular}

TABLA 12.

Distribución de artículos según Enfoque Terapéutico en la Revista Internacional de Psicología Clínica y de la Salud entre 2004 y 2010

\begin{tabular}{lcccccccc}
\hline \multicolumn{1}{c}{ Enfoque Terapéutico } & 2004 & 2005 & 2006 & 2007 & 2008 & 2009 & 2010 & Total \\
\hline Enfoque Psicoanalítica & 0 & 0 & 0 & 0 & 0 & 0 & 0 & 0 \\
Enfoque Cognitivo conductual & 4 & 6 & 4 & 4 & 5 & 4 & 2 & 29 \\
Enfoque Humanista & 0 & 0 & 0 & 0 & 0 & 0 & 0 & 0 \\
Enfoque Sistémico & 0 & 0 & 0 & 0 & 0 & 0 & 0 & 0 \\
Total & 4 & 6 & 4 & 4 & 5 & 4 & 2 & 29 \\
\hline
\end{tabular}

\section{DISCUSIÓN}

Es importante señalar que el objetivo del presente estudio no es evaluar la calidad de las revistas consideradas, si no, realizar una revisión y evaluación de las directrices y tendencias de los artículos de psicología clínica en Chile, Latinoamérica y España a través de un estudio bibliométrico entre los años 2004 y 2010, realizando un análisis en cuanto a la cantidad de artículos publicados, el índice de autoría, el área de conocimiento que abordan y los principales enfoques terapéuticos.

Respecto a la cantidad de artículos publicados en cada una de las revistas es posible evidenciar un menor número en 
Terapia Psicológica, lo que se explica al ser una revista semestral a diferencia de la Revista Latinoamericana de Psicología y Revista Internacional de Psicología Clinica y de la Salud que cuentan con tres números al año. Cabe destacar que en las tres revistas analizadas existe un notorio aumento de artículos publicados en los años 2007 y 2008 lo cual según autores como Alcain y Lascurain (2002) se explica por un aumento de la actividad científica y tecnológica que se realizó en esos años, principalmente en América Latina.

Un dato importante a señalar, es que la Revista Latinoamericana de Psicología acepta trabajos de diversos campos y áreas de la psicología por lo que debió realizarse un análisis exhaustivo para obtener sólo artículos del área clínica, realizado mediante dos profesionales, llegando a un $92 \%$ de acuerdo en la inclusión de los artículos.

Respecto al índice de autoría existen diferencias, ya que la revista Terapia Psicológica muestra una mayor inclinación hacia autorías individuales 0 de dos personas, la Revista Latinoamericana de Psicología en autorías de dos 0 tres personas y la Revista Internacional de Psicología Clinica y de la Salud presentan una tendencia a publicaciones con tres y cuatro autores, lo cual puede ser bastante positivo en cuanto a que se habla de colaboración y fortalecimiento de grupos de trabajo. Se destaca además que en las tres revistas se encuentran artículos con un número elevado de autores ( 6 a 10) lo cual genera atención frente a la dinámica de trabajo grupal abordada y a los consensos obtenidos en ellos.

Frente al análisis de los principales intereses académicos es posible señalar que la Revista Latinoamericana de Psicología y la Revista Internacional de Psicología Clínica y de la Salud se concentran en la psicopatología; evaluación psicológica y la prevención y promoción de la salud, prestando una baja atención en el diagnóstico psicológico y en la persona del terapeuta. Diferente es el caso de la revista Terapia Psicológica ya que los intereses académicos están en la evaluación psicológica y la intervención/psicoterapia. Se visualiza también que a pesar de tener pocos estudios, la persona del terapeuta goza de mayor atención que en las otras revistas.

Para el caso de los enfoques psicológicos, los artículos de estas tres zonas se presentan de forma similar, ya que el mayor número de publicaciones es el enfoque cognitivo conductual tanto en la Revista Terapia Psicológica, Revista Latinoamericana de Psicología y Revista Internacional de Psicología Clinica y de la Salud. Cabe señalar que en estas revistas no se encontraron artículos con un enfoque humanista y en una escasa representatividad se encuentran artículos con un enfoque sistémico o psicoanalítico.

Estos datos señalan que en Iberoamérica existe una marcada tendencia a realizar artículos científicos desde modelos conductuales 0 cognitivos conductuales, lo cual podría deberse a que este enfoque se complace de obtener gran respaldo teórico para muchos trastornos mentales, además de que existe una fuerte tendencia en evaluar la efectividad de sus tratamientos psicológicos.

Frente a estos resultados se puede apreciar que en estas zonas continúa un predominio por temas de la psicología clínica como la evaluación y la psicopatología, a diferencia de temáticas como la persona del terapeuta, los cuales aun no se potencian ni exploran de forma extensa, pese a que en los últimos años este tema ha cobrado gran relevancia en la preparación de la formación tanto de pregrado como de postgrado (Rey et al. 2009).

Es posible señalar que en vista de los resultados obtenidos del análisis de estas 
tres revistas, se observan diferencias comprensibles, dada la diversidad de las zonas, y estas pueden ser un reflejo de los distintos niveles de desarrollo que tienen, lo cual no sólo está relacionado con el tamaño de la población sino también al grado de desarrollo de la ciencia y tecnología del lugar (Alcain y Lascurain, 2002).

Dentro de las limitaciones que presenta el estudio, se considera que no analiza ni da cuenta del índice de colaboración entre países, ya que existen revistas que dan cabida a trabajos nacionales, mientras que para otras revistas el criterio a utilizar consiste en obtener un mayor número de colaboradores extranjeros.

Otro aspecto que no se consideró es el idioma aceptado por la revista para los artículos científicos, ya que no será igual la producción ni contribución cuando se publica solo en un idioma que cuando se permite publicar en dos o más idiomas.

Finalmente, cabe señalar que el presente estudio busca identificar las diferencias y similitudes de las distintas revistas y publicaciones, siempre con una visión constructiva que permita analizar otros puntos de vista y que permita generar un mayor nivel de exigencias dentro de la región.

\section{REFERENCIAS}

Aceituno, R., Krause, M. y Winkler, M. (2000). Dialogo acerca de la investigación en Psicología Clínica. Terapia Psicológica, VII (5), 7-19.

Agudelo, D., Bretón López, J. y Buela-Casal, G. (2003). Análisis bibliométrico de las revistas de Psicología Clínica editadas en castellano. Psicothema, 15, 507-516.

Alcain, M. D. y Carpintero, H. (2001). La Psicología en España a través de revistas internacionales: 1981-1999. Papeles del Psicólogo, 78, 11-20.

Alcain, M. D. y Lascurain, M. L. (2002). Análisis de las revistas latinoamericanas de psicología incluidas en el "Directorio
Latindex". Papeles del Psicólogo, 83, 3443.

Buela-Casal, G. (2001). La psicología española y su proyección internacional. El problema del criterio: internacional, calidad y castellano $y / 0$ inglés. Papeles del Psicólogo, 79, 53-57.

Buela-Casal, G. (2002). La evaluación de la investigación científica: el criterio de la opinión de la mayoría, el factor de impacto, el factor de prestigio y "Los Diez Mandamientos" para incrementar las citas. Análisis y Modificación de Conducta, 28, 455-475.

Buela-Casal, G. (2003). Evaluación de la calidad de los artículos y de las revistas científicas: Propuesta del factor impacto ponderado y de un índice de calidad. Psicothema, 15, 23-35.

Buela-Casal, G., Carretero Dios, H. y De los Santos Roig, M. (2002). Estudio comparativo de las revistas de Psicología en castellano con factor de impacto. Psicothema, 14, 837-852.

Buela-Casal, G. y López, W. (2005). Evaluación de las revistas científicas Iberoamericanas de Psicología. Iniciativas y estado actual. Revista Latinoamericana de Psicología, 37, 211-217.

Buela-Casal, G., Sierra, J.C., Carretero-Dios, H. y De los Santos-Roig, M. (2002). Situación actual de la evaluación psicológica en lengua castellana. Papeles del Psicólogo, 83, 27-33.

Calvache, O., López, W. y Mayorga, E. (2002). Psicología Clínica: 20 años de la Revista Avances en Psicología Clínica Latinoamericana. Avances en Psicología Clínica Latinoamericana, 30, 401-427.

Civera, C. y Tortosa, F. (2001). Estado de la Investigación Psicológica en España: el grado de doctor y la investigación académica (1976-1998). Papeles del Psicólogo, 79, 42-52.

Jiménez-Contreras, E., Faba, C. y Moya, A. (2001). El destino de las revistas científicas nacionales. El caso español a través de una muestra (1950-90). Revista Española de Documentación Científica, 24, 147-161.

Montero, I. y León, O. (2007). A guide for naming research studies in Psychology. International Journal of Clinical and Health Psychology, 7, 847-862.

Moyano, E. y Ramos, N. (2000). Contexto y evaluación de la investigación psicológica 
en el Chile de fin de siglo. Psykhe, 9, 6375.

Ortiz, J. y Vera-Villarroel, P. (2003). Investigaciones en psicología clínica basadas en la evidencia en Chile: un análisis bibliométrico de tres revistas de psicología. Terapia Psicológica, 21, 61-66.

Pelechano, V. (2002). Valoración de la actividad científica en psicología? pseudoproblema, sociologismo 0 idealismo?. Análisis y Modificación de Conducta, 28, 323-362.

Perdomo, S., Zambrano, S., López, W. y Pérez, A. (2003). Veinte años de investigación en psicología en la Universidad Católica de Colombia: Análisis bibliométrico de la investigación publicada. Acta Colombiana de Psicología, 9, 105-125.

Pérez, M. (2002). La psicología en el contexto de la cultura española. Análisis y Modificación de Conducta, 28, 405-430.

Rey, C., Martínez, J. y Guerrero, S. (2009). Tendencia de los Artículos en Psicología Clínica en Iberoamérica. Terapia Psicológica, 27, 61-71.

Vera-Villarroel, P. y Lillo, S. (2006). La investigación actual en Psicología Clínica en Chile: Un análisis a partir de la producción. Terapia Psicológica, 24, 221230. 